\title{
CONTROL DE UN BRAZO ROBÓTICO ANTROPOMÓRFICO CON 6 GRADOS DE LIBERTAD TELEOPERADO PARA LA SEGURIDAD INDUSTRIAL EN EL PERÚ
}

\section{CONTROL OF A REMOTELY CONTROLLED ANTHROPOMORPHIC ROBOTIC ARM WITH SIX DEGREES OF FREEDOM TO ENHANCE INDUSTRIAL SECURITY IN PERU}

\author{
Elmer Javier Córdova Zapata', \\ Hans C. Rojas Caytuiro², Eduardo Espinoza Marazo ${ }^{2}$ y Jerson Herrera Yui ${ }^{2}$
}

\begin{abstract}
RESUMEN
En este proyecto de investigación se propone una solución a la manipulación directa de los materiales peligrosos utilizados en las industrias químicas o nucleares. Este proyecto describe el diseño de un brazo robótico de 06 grados de libertad controlado de forma remota mediante una interfaz humana utilizando una variedad de sensores en el brazo del operador y la transmisión bluetooth. La interfaz humana es un guante de datos mejorado que cuenta con 5 sensores de flexión y una IMU para la rotación. Un PIC16F877A con una interfaz bluetooth maestro envía todos los datos a través de bluetooth para el esclavo, el brazo robótico. Un Arduino instalado en el brazo robótico procesa los datos recibidos y consigue mover los servomotores en consecuencia.
\end{abstract}

Palabras clave: PIC, Arduino, Bluetooth, Conversor ADC, Interfaz Humana, Flex Sensor.

\begin{abstract}
This project proposes a solution to direct handling of hazardous materials in the chemical and nuclear industries: A six-degree of freedom robotic arm remotely controlled by a human interface using a variety of sensors in the operator arm and bluetooth transmission. The human interface is an enhanced data glove with 5 sensors for flexion and one IMU for rotation. A PIC16F877A with a bluetooth interface master sends all data through bluetooth to the slave; that is, the robotic arm. An Arduino installed in the arm processes data received and move servomotors accordingly.
\end{abstract}

Keywords: PIC, Arduino, Bluetooth, ADC Conversor, Human Interface, Flex Sensor.

1 Posdoctorado en Ciencias de la Educación, Doctor en Ciencias de la Educación, Maestro en Ciencias de la Electrónica con mención en Control y Automatización, Ingeniero Mecatrónico, Director de la Escuela Profesional de Ingeniería Mecatrónica, Facultad de Ingeniería, Universidad Ricardo Palma. <ejcordova5@hotmail.com>

2 Co-autores y egresados de la Escuela Profesional de Ingeniería Mecatrónica de la Universidad Ricardo Palma: Hans C. Rojas Caytuiro, <ngineer_hans@hotmail.com>; Eduardo Espinoza Marazo, <noduarzo@outlook.com> y Jerson Herrera Yui, <jerson_hy@hotmail.com> 


\section{INTRODUCCIÓN}

Actualmente, las industrias generan residuos altamente tóxicos. Tal es el caso de las industrias químicas o las que trabajan con material radiactivo. Estos materiales son muy peligrosos por sus propiedades de corrosión o radiación que generan un daño irreparable al operario.

Según el análisis de las estadísticas del SINC (Servicio de Información y Noticias Científicas) [1], el $22 \%$ de los trabajadores en Europa inhalan humos y vapores, al menos, durante una cuarta parte de su vida laboral y, solo en España, más del 17\% de los profesionales españoles manipula contaminantes químicos en su trabajo. Mientras se trabaja, la inhalación es la manera más común de absorber sustancias neurotóxicas.

Las operaciones de inspección y mantenimiento de las zonas de trabajo en una industria, por lo general, son largas y costosas, además de realizarse manualmente. La exposición de los operadores a la radiación sería mayor; ello es un factor crítico que, junto con el elevado coste que supone una interrupción temporal en el funcionamiento del sistema en cuestión, justifica, sin lugar a dudas, la utilización de sistemas robotizados, normalmente teleoperados, total o parcialmente, que sustituyan al operador. [2]

Utilizando un robot, los operarios pueden mantenerse en el proceso laboral durante más tiempo sin sufrir daños físicos o cansancio a causa de los materiales peligros, ya que el operario no tendrá intervención alguna en su manipulación. Existen 2 opciones con las cuales se puede desarrollar un sistema: una de ellas es utilizando manipuladores con unión mecánica aislada a través de un cristal protector, donde el operario puede hacer un seguimiento directo del proceso. Sin embargo, tienen sus limitaciones, ya que al ser mecánicos en su totalidad tienen cierto grado de dificultad en su manejo y movimientos limitados. La segunda opción es utilizar sistemas con mando remoto por radio o cable. Estos manipuladores nos permiten la flexibilidad necesaria para controlar elementos de peso variable y forma no definida, más aún en el caso de trabajar con materiales de alto grado de contaminación. Es preciso considerar la importancia que tiene el espacio ocupado por los materiales o residuos radiactivos, ya que para el control de estos solo se podrá lograr si tienen dimensiones adecuadas con las que un operario pueda controlar con sus propias manos, ya que no se pretende utilizar este sistema para materiales demasiado pesados o de gran volumen.

Teniendo en cuenta esto, el presente proyecto trata sobre el desarrollo de un brazo robótico antropomórfico, el cual se podrá utilizar para transmitir un movimiento similar al operario que lo manipule remotamente, por lo que se trata de un sistema de asistencia ideal. Para este proyecto se utilizó la impresión 3D, la cual nos dio la capacidad de hacer las piezas que conforman el brazo a medidas más precisas, según lo diseñado en el software Solidworks. Adicionalmente, se utilizó un microcontrolador PIC 16f877a para lecturas análogas de los sensores, el cual es muy barato y confiable, y, para la recepción de datos, un arduino, por lo que no fue necesario utilizar otro controlador como un PLC, ya que incrementarían notablemente el costo del robot.

\section{OBJETIVOS}

- Controlar materiales peligrosos a distancia, de forma que el robot se mueve de la misma manera que la mano del controlador.

- Diseñar e implementar un sistema de seguridad industrial que esté al alcance de la pequeña y mediana empresa. 


\section{MÉTODO}

Se utilizó un método científico cuantitativo de investigación cuyo desarrollo del proyecto de investigación es el siguiente:

Se utilizó el algoritmo de Denavit-Hartenberg para hallar la cinemática directa del brazo robótico y se realizó el diagrama de hilos, como se muestra en la Figura1.

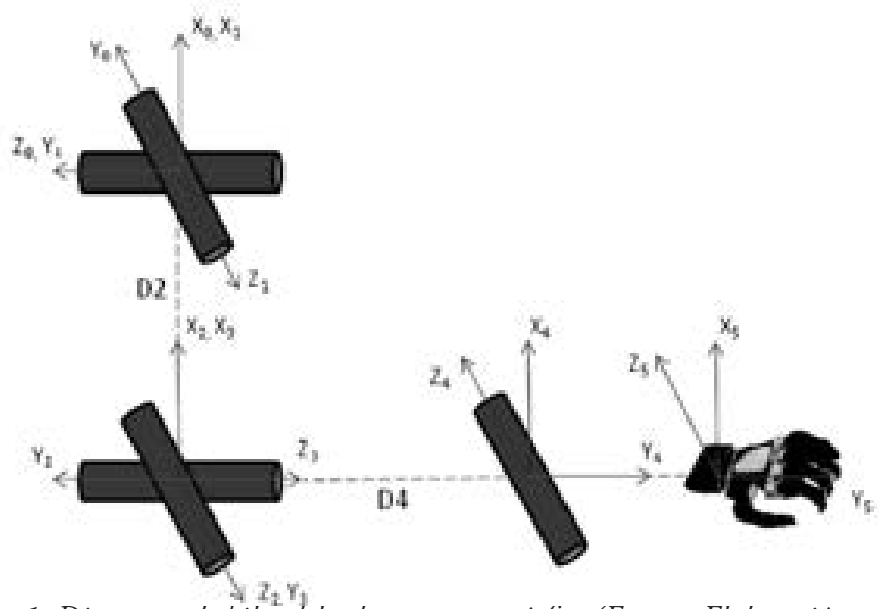

Figura 1. Diagrama de hilos del robot antropomórfico (Fuente: Elaboración propia)

Luego, se obtuvo la tabla I, en la cual se pondrán todos los parámetros del robot.

TABLA I. PARÁMETROS DEL ROBOT

\begin{tabular}{|c|c|c|c|c|}
\hline i & $\theta i$ & di & ai & $\alpha i$ \\
\hline 1 & $(\theta) 7$ & 0 & O & $+p i / 2$ \\
\hline 2 & & 0 & $D_{2}$ & 0 \\
\hline 3 & $\theta$ & 0 & 0 & $+p i / 2$ \\
\hline 4 & & $D_{4}$ & O & $+p i / 2$ \\
\hline
\end{tabular}

Con los valores obtenidos de la Tabla 1, se reemplazó los valores en una matriz de DenavitHartenberg (1), diferente para cada una de las articulaciones.

$$
A_{i-1}^{i}=\left|\begin{array}{cccc}
\cos \left(\theta_{i}\right) & -\sin \left(\theta_{i}\right) \cos \left(\alpha_{i}\right) & \sin \left(\theta_{i}\right) \sin \left(\alpha_{i}\right) & \cos \left(\theta_{i}\right) a_{i} \\
\sin \left(\theta_{i}\right) & \cos \left(\theta_{i}\right) \cos \left(\alpha_{i}\right) & -\cos \left(\theta_{i}\right) \sin \left(\alpha_{i}\right) & \sin \left(\theta_{i}\right) a_{i} \\
0 & \sin \left(\alpha_{i}\right) & \cos \left(\alpha_{i}\right) & \alpha_{i} \\
0 & 0 & 0 & 1
\end{array}\right|
$$


Finalmente, para obtener la cinemática directa, multiplicamos las 4 matrices de DenavitHartenberg (2) y obtenemos una matriz de transformación final, la cual nos proporcionará la cinemática directa del robot.

$$
{ }^{0} T_{4}={ }^{0} T_{1}+{ }^{1} T_{2} \cdot{ }^{2} T_{3}+{ }^{3} T_{4}
$$

\section{Diseño mecánico}

El diseño mecánico se desarrolló usando el software Solidworks, el cual se logró diseñar y simular. Se utilizó una impresora 3D con el material $\mathrm{ABS}$, que es un plástico que nos permitirá trabajar con las piezas en físico. Se utiliza ABS, ya que este plástico es resistente y barato, además, si hubiese imperfecciones, se puede pulir fácilmente, Figura 2.[5]

La mano derecha está formada por 17 articulaciones unidas por tornillos de 1/8 de pulgada de diámetro (Figura 3), estas medidas son dadas aproximadas para tener el tamaño promedio de los objetos a manipular, lo que puede variar dependiendo de qué tipo de objetos vamos a manipular [6]. Las articulaciones actúan con sistemas de cableado de nylon de $0.8 \mathrm{~mm}$ de diámetro. Se usa nylon de este grosor, ya que como es más grueso, tiene menor deformación al ser sometido

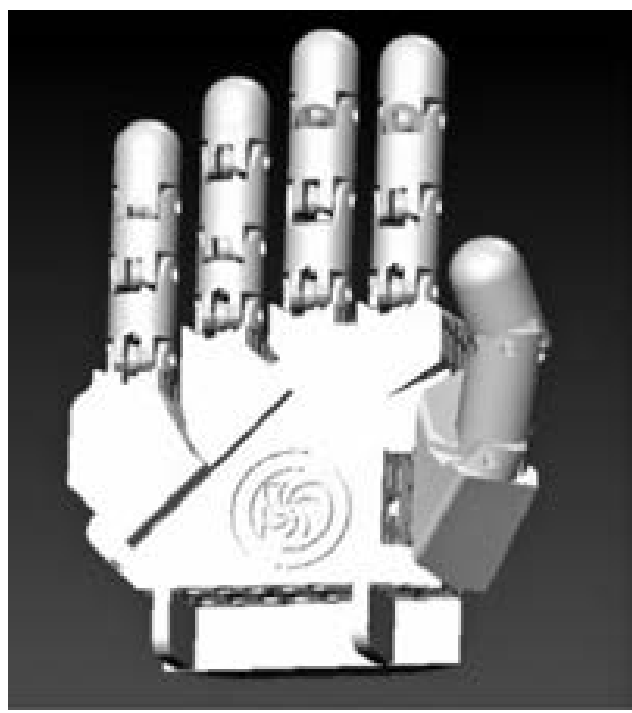

Figura 2. Diseño de la mano (Fuente: Elaboración propia) a esfuerzos. Estos hilos de nylon se introducen en los canales de los dedos. De esta forma, les dan la capacidad de contraer y expandir los dedos y la mano, al jalar cada nylon desde su respectiva vía [7].

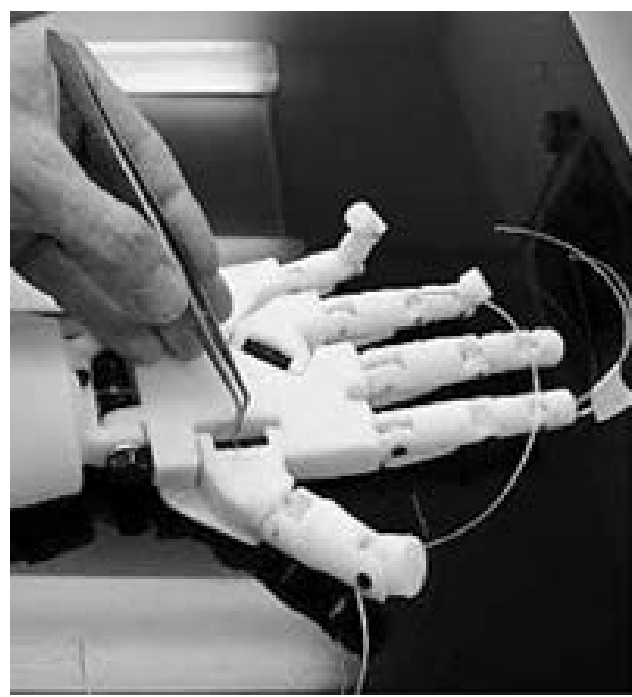

Figura 3. Cables de nylon para mover las articulaciones (Fuente: Inmoov 2010)

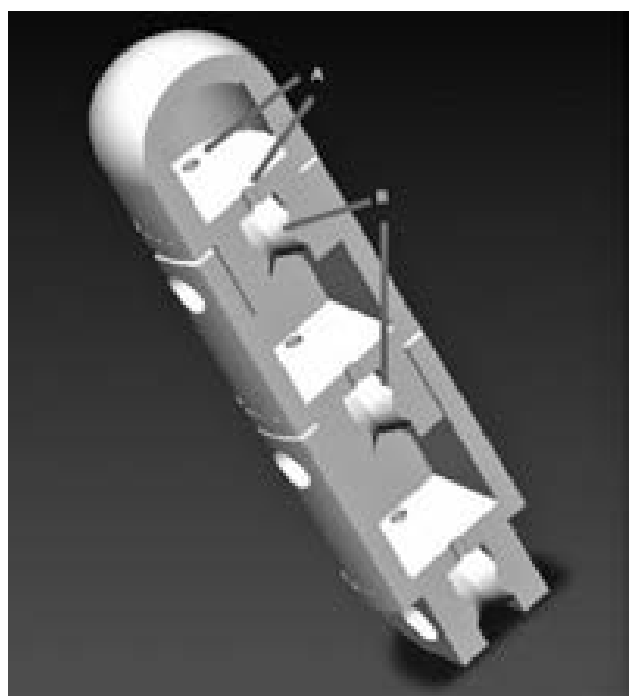

Figura 4. Vista de sección de un dedo. (Fuente: Elaboración propia) 
Se puede ver, en la Figura 4, la vista de sección de un dedo, donde, en la zona "A", se aprecian los orificios por donde atraviesa el nylon, y, en la zona "B", se observan las roldanas, diseńadas para que el cable pueda pasar por un camino guiado.

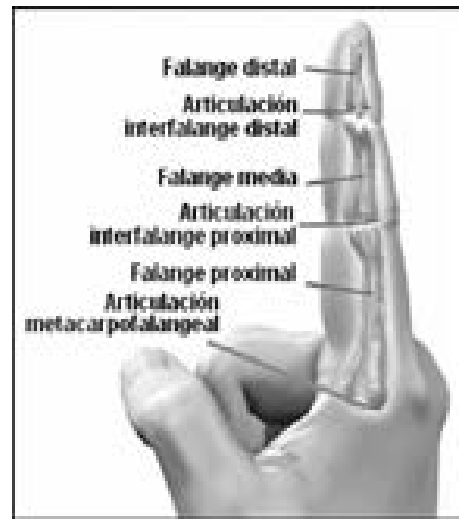

Figura 5. La mano humana y sus articulaciones. (Fuente: Robots-Argentina)
TABLA II. RANGO DE MOVILIDAD DE UN DEDO HUMANO [8]

\begin{tabular}{|c|c|c|c|}
\hline GDL & Articulación Humano & Estándar (०) & $\theta$ \\
\hline 1 & Metacarpofalangeal (AMF) & $90^{\circ}$ & $\theta 1$ \\
\hline 2 & Interfalange proximal (AIP) & $110^{\circ}$ & $\theta 2$ \\
\hline 3 & Interfalange distal (AID) & $60-70^{\circ}$ & $\theta 3$ \\
\hline
\end{tabular}

Fuente: Elaboración propia

La parte del antebrazo se une a la mano por la muñeca. El antebrazo tiene en su interior los seis servomotores, cinco que permitirán el movimiento del nylon y los dedos, y uno que estará situado al final para darle el movimiento de giro al brazo. Se puede apreciar la distribución de los servomotores con sus respectivos anillos que permitirán que, con un solo servomotor, se pueda mover cada nylon en su respectiva vía (Figura 6).

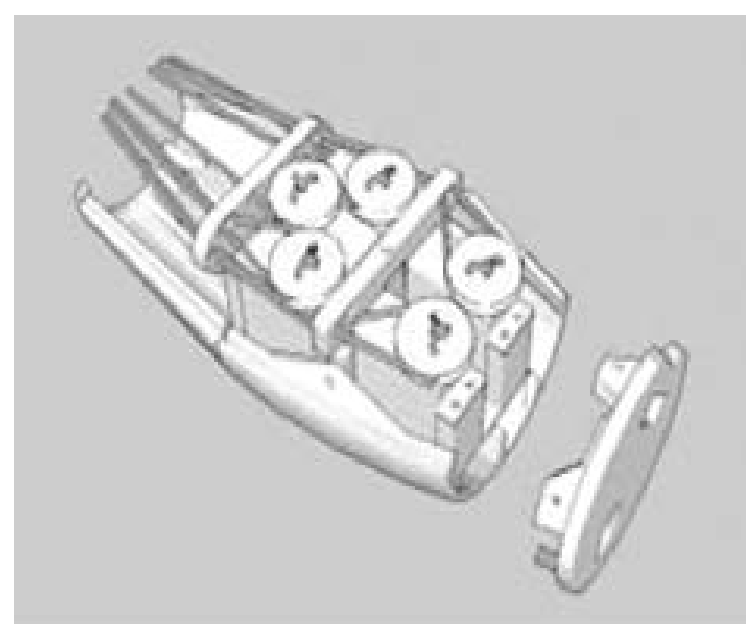

Figura 6. Ensamblaje de los servomotores (Fuente: Elaboración propia)

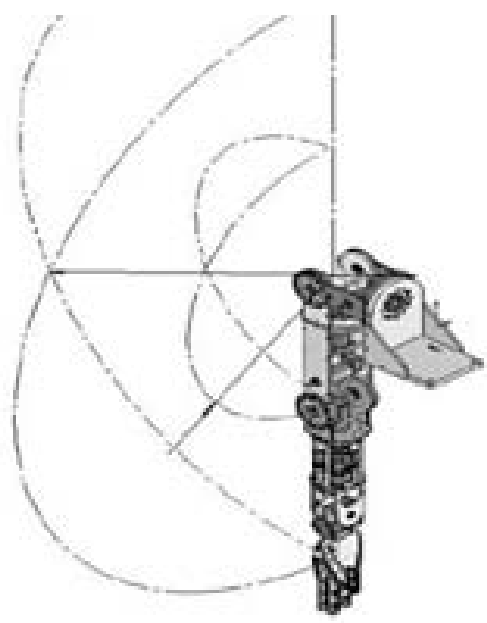

Figura 7. Proyección final del brazo robótico Vista isométrica (Fuente: Cenidet)

Para emular los movimientos del codo, se necesitará un servomotor que hará también el movimiento de flexión. La zona más crítica y que necesita mayor torque es la zona del hombro [15], que, para poder realizar movimientos, necesitará el uso de 2 servomotores.

El brazo entero se basa en un sistema, como se observan en la Figura 7, además de luego estar incorporado en una base para apoyarse [9]. 


\section{Diseño electrónico}

Se diseńó un guante de datos, que tiene incorporado sensores de flexión y un IMU con comunicación I2c.

Se eligió utilizar 1 flex sensor por cada dedo, ya que estos están diseñados para medir flexión de movimientos corporales. Estos son sensores resistivos, es decir, que cambian su resistencia a medida que este se flexiona. Esto se aprecia en la Figura 8. [10]

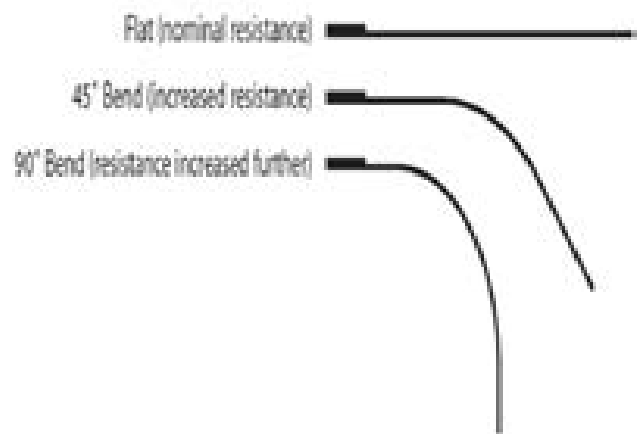

Figura 8. Cambio de resistencia con Flexión (Fuente: Datasheet flexsensor)

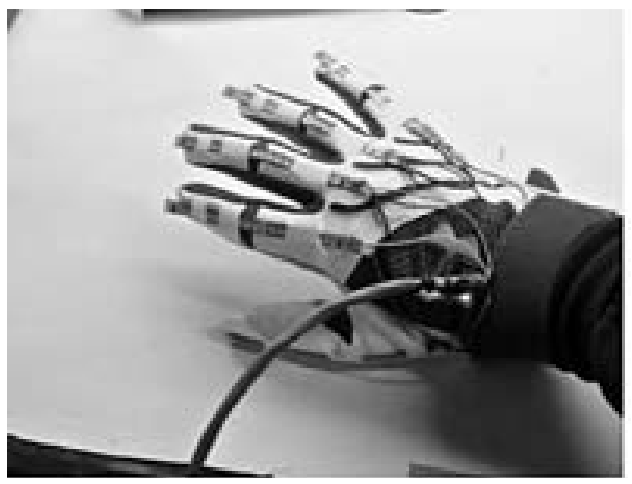

Figura 9. Guante con Flex sensor (Fuente: Benkras)

Este sensor es el que precisamente se necesita para medir flexiones de un dedo. Estos sensores estarán en el guante, como se muestra en la Figura 9.

Se optó por usar un divisor de tensión para obtener un voltaje útil. Para el cálculo de la resistencia de pull-up, se usó la fórmula 3, que maximiza la variación de voltaje del divisor de tensión:

$$
R s=\sqrt{R \max +R \min }
$$

Cada sensor de flexión es único en su rango de resistencia, por lo que cada sensor tendrá su propia resistencia de pull-up [11]. En la Tabla III, se muestran los valores de resistencia mínimo, máximo y resistencia de divisor de tensión.

TABLA III. CÁLCULO DE RESISTENCIA DE PULL-UP

\begin{tabular}{|c|c|c|c|}
\hline Flex Sensor & $\operatorname{Rmin}(\mathrm{K})$ & $\operatorname{Rmax}(\mathrm{K})$ & $\mathrm{Rs}(\mathrm{K})$ \\
\hline 1 & 8.61 & 15.00 & 12 \\
\hline 2 & 9.48 & 18.20 & 12 \\
\hline 3 & 10.03 & 21.30 & 15 \\
\hline 4 & 10.67 & 21.50 & 15 \\
\hline 5 & 8.89 & 13.40 & 10 \\
\hline
\end{tabular}

(Fuente: Elaboración propia)

El diseño del circuito de acondicionamiento del sensor de flexión se muestra en la Figura 10. Consta de un divisor de tensión para la obtención de un voltaje y arreglos de amplificadores operacionales para el acople de impedancia, resta de offset y amplificación de voltaje [12]. 


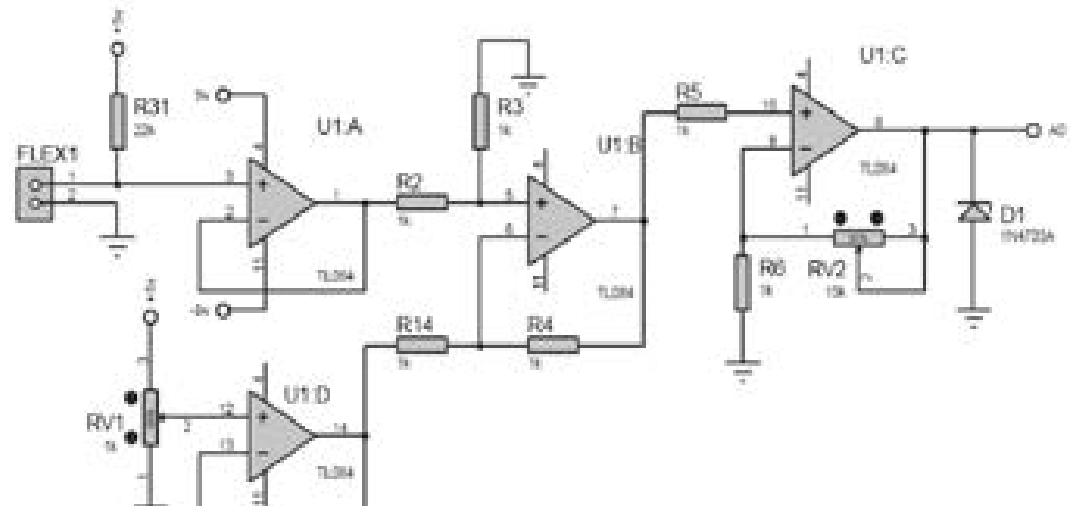

Figura 10. Circuito para el Flex Sensor (Fuente: Elaboración Propia)

\section{Comunicación vía Bluetooth:}

Los módulos bluetooth HC-05 maestro y HC-06 esclavo son dispositivos que implementan comunicación serial sin necesidad de cableado. El dispositivo es muy pequeño, consume $8 \mathrm{~mA}$ de corriente, trabaja con lógica TTL positiva y posee un alcance de 10 metros. Estos módulos poseen solo 4 pines: $+5 \mathrm{~V}$, GND, Tx y Rx como se muestra en la Figura 11. [3]

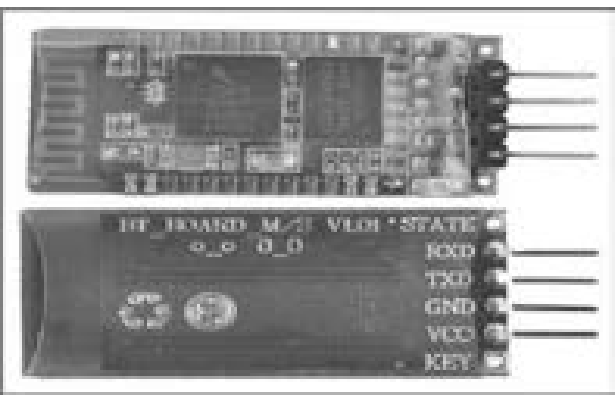

Figura 11. Módulo Bluetooth HC-06 (Fuente: SparkFun)

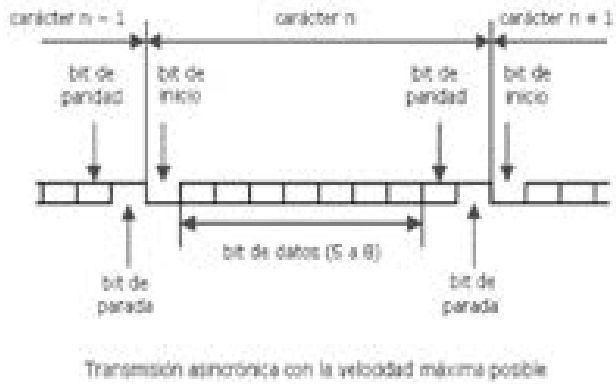

Figura 12. Transmisión serial continúa (Fuente:Sparkfun)

Estos dispositivos se usaron por la baja interferencia que poseen y su alcance de $10 \mathrm{~m}$, que es suficiente, ya que el operario maneja los materiales de cerca. La velocidad de comunicación es de suma importancia para que el PIC16F877A pueda mandar los datos correspondientes de manera más rápida [4]. Para este proyecto, se configuró la velocidad de transmisión a 56700 BPS. Se usará un módulo Bluetooth maestro HC-05 para la interfaz humana, es decir, el guante y un módulo esclavo HC-06 para el robot antropomórfico. De esta manera, el operario que controla el guante tendrá control en tiempo real sobre el robot antropomórfico.

La transmisión es de forma serial RS232 con lógica TTL positiva, como se muestra en la Figura 12.

\section{Programación}

La lógica de la programación que se siguió en el presente proyecto consta de una planta controlada a distancia. Esto significa que se están usando dos microcontroladores y dos programas diferentes: el 
esclavo y el maestro. La programación del micro controlador maestro, el PIC, es de la siguiente manera: consta de 5 flex sensor y un MPU6050, en total 6 datos. El PIC solo se encarga de leer los datos analógicos y de I2c para enviarlos como están al micro controlador esclavo Arduino.

Para los ADC, se escogió una resolución de 8 bits, ya que es más manejable a la hora de mandar esta información por bluetooth de forma serial. Se optó por sacar un promedio de estas señales, para luego mandar cada dato separado por comas. Una rutina de código para enviar 5 datos se muestra en la Figura 13.

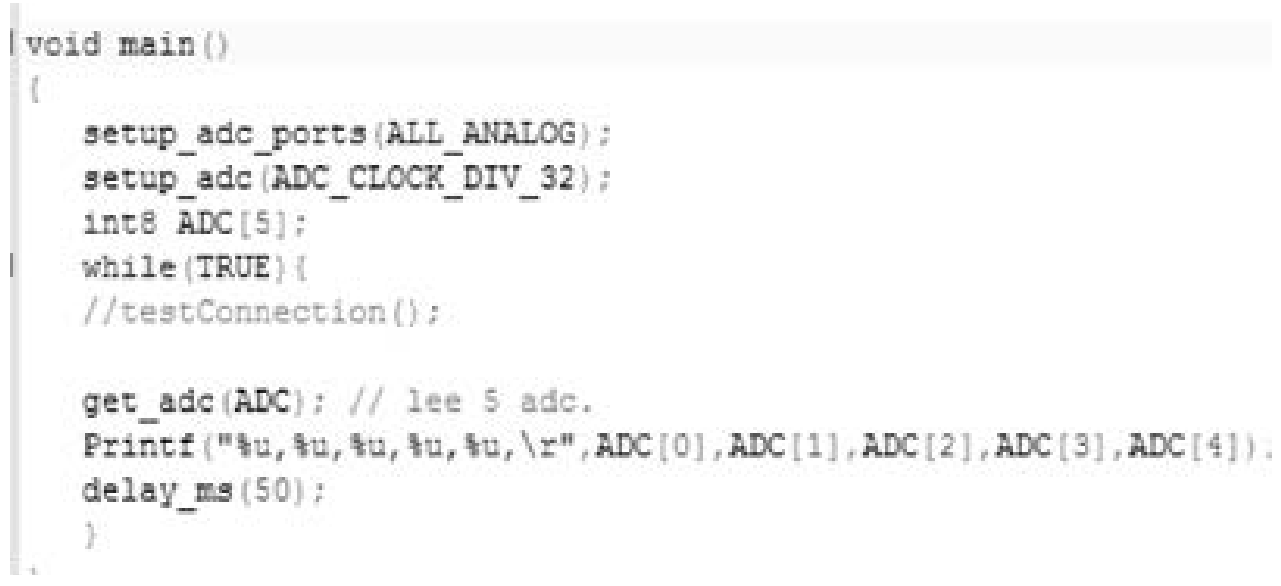

Figura 13. Códigos de programación (Fuente: Elaboración propia)

La subrutina get_adc (ADC) compila en un vector hasta $5 \mathrm{ADC}$, elimina el mínimo, el máximo y calcula el promedio. Esto lo hace para los 5 ADC a enviar [13]. Este código es muy fácil de mantener y modificar para 8 ADC. El micro controlador esclavo Arduino [14] debe poder interpretar estos datos en formato de caracteres y almacenarlos también en un vector. El bucle principal del programa en Arduino para el movimiento de cinco dedos se muestra en la Figura 14.

La sub función get_data() lee de la comunicación serial letra por letra para formar los números, y empieza con el segundo numero cuando encuentra una coma. Se tiene también una subrutina dentro de get_data() para el depurado del código y el correcto funcionamiento de los motores. Este depurado se hace directo de una

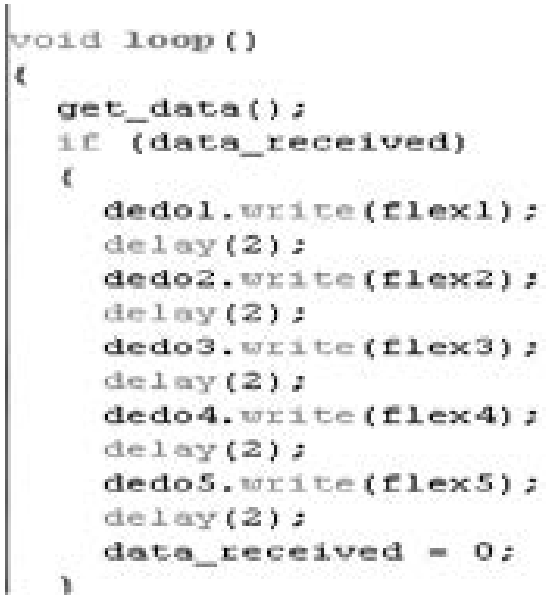

Figura 14. Programación de los dedos (Fuente: Elaboración propia) computadora con el Serial Monitor del software de Arduino para controlar la posición de todos los servos (ej. 255, 255, 255, 255, 255 cierra la mano donde $255=5 \mathrm{~V}$ en el ADC) o de uno solo (ej. A90 cierra el dedo meńique $90^{\circ}$ con respecto a la palma). [15]

Ambas programaciones son de fácil mantenimiento y fácilmente ampliables a más datos y están en lazo abierto. Se pueden usar diversos sensores para la realimentación de los motores e incorporarlos al código. 


\section{RESULTADOS}

La respuesta del sensor para una entrada escalón [16], cierre rápido del guante, se muestra en la Figura 15.

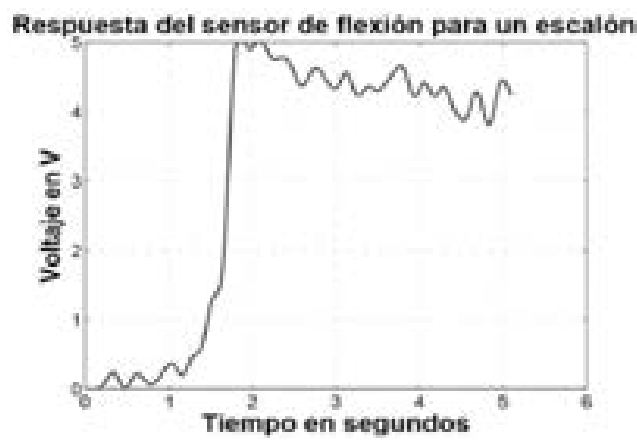

Figura 15. Respuesta al escalón del sensor de flexión. (Fuente: Elaboración propia)

Se debe de tener una protección física contra estrés en las borneras del sensor de flexión, ya que estas son delicadas. Esto alargará la vida útil de los sensores.

Se notó que la resistencia del sensor de flexión decrece con su uso.

Se obtuvo el procesamiento de seńal por software de 8 señales análogas. Estas permitieron el estudio del movimiento del brazo humano para poder controlar un brazo robótico antropomórfico.

Se observa, en la Figura 16, que el serial monitor del Arduino ayuda a verificar el movimiento de los servos en los dedos. De ambas formas, un dedo o todos a la vez [17].

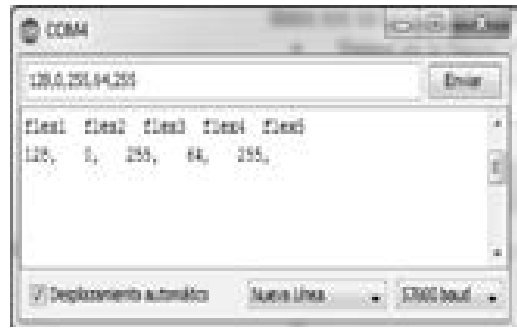

Figura 16. Serial Monitor del Arduino para distintos comandos (Fuente: Elaboración propia)

Finalmente, en la Figura 17, se muestran imágenes del proyecto en su estado final:
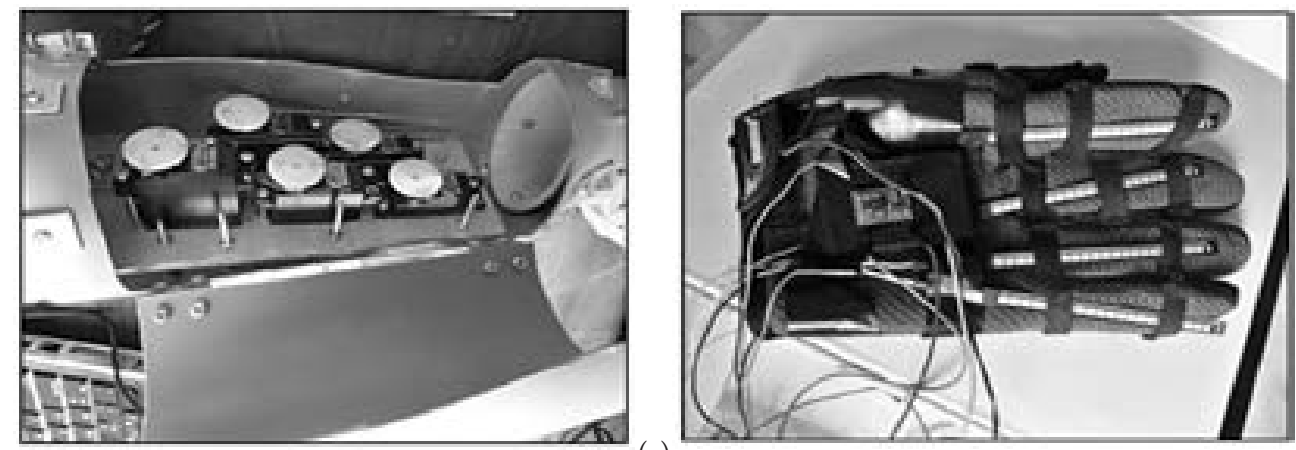

(a) 


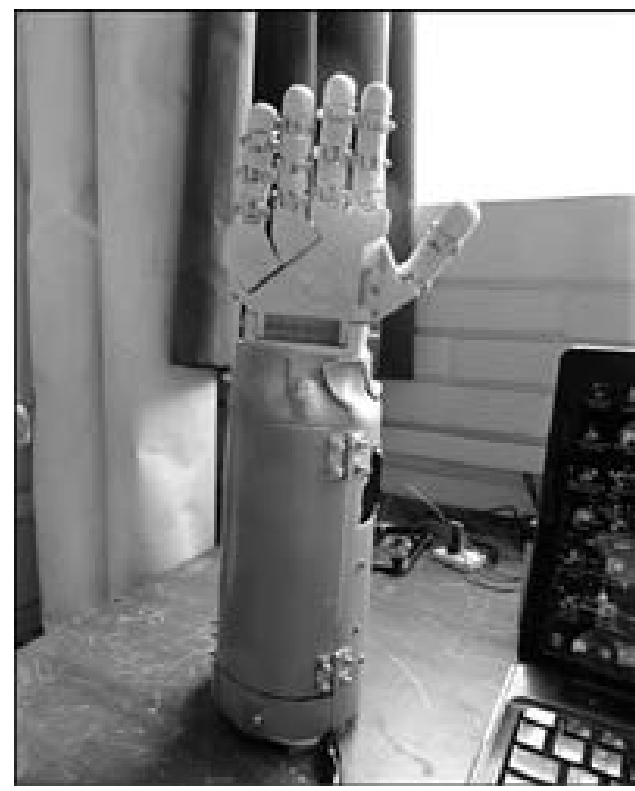

(b)

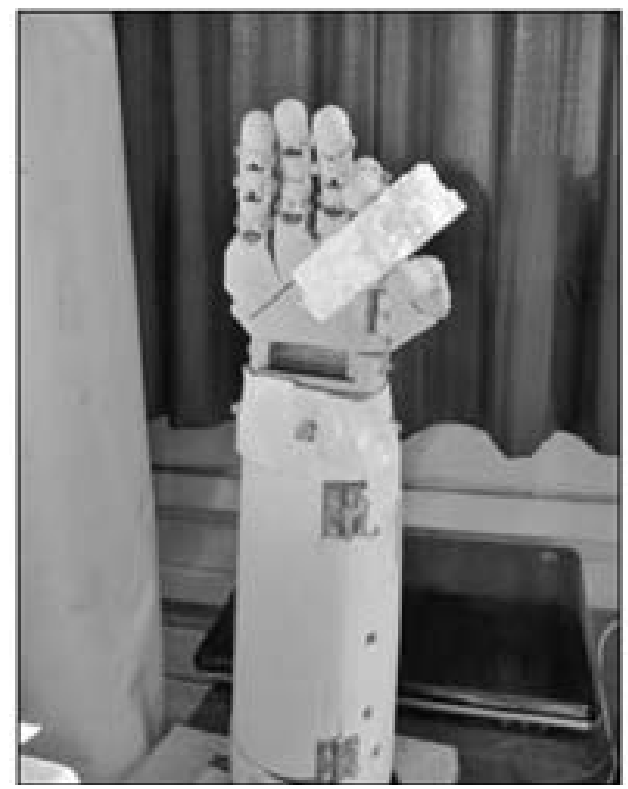

Figura 17. Resultado final de brazo robótico (Fuente: Elaboración propia)

\section{DISCUSIÓN}

Se consiguió emular el movimiento de una mano humana, la cual es manipulada a través del guante de datos con un tiempo de respuesta de 198 ms. Además se utilizaron los sensores de flexión con un adecuado sistema de acondicionamiento trabajando en su zona activa de $55.37 \mathrm{~mm}$ para una longitud total de $73.66 \mathrm{~mm}$ con una potencia nominal de 0.5 vatios continuos.

La velocidad de transmisión de datos se escogió para un control suave y continuo de los servomotores. Si bien esta velocidad no afecta el delay entre envío de datos y la obtención de estos, la velocidad de transmisión afecta el ancho de trama drásticamente. Para 57600 BPS, se obtuvo un total de $3.55 \mathrm{mSeg}$ por trama a 5 datos de 1 byte cada uno. Al ir aumentando los datos a enviar, también se deberá aumentar la velocidad de transmisión.

Se puede adicionar más sensores para el movimiento del codo y del hombro, que permitirían tener más precisión, además de sensores de fuerza resistidos (FSR), que medirían la fuerza con la que se estaría presionando el brazo para que este no llegue a dańar el material que estuviese agarrando y le darían un límite al coger objetos.

\section{Observaciones}

El rango de movimiento de $180^{\circ}$ de los servomotores excedía en algunos casos los límites de las articulaciones.

Si se decide usar articulaciones adicionales en la mano, se lograría una respuesta más precisa; sin embargo, esto encarecería y complicaría el diseño. Se decidió usar servomotores de $10 \mathrm{~kg}$-cm con nylon, ya que, si se usarán micro-servos para las articulaciones, estos no tendrían el torque suficiente para mover la mano. 
Las tolerancias en el diseño deben ser pequeñas, debido a que este sistema es muy compacto y puede haber colisión de piezas.

Se debe tener una protección física contra estrés en las borneras del sensor de flexión, ya que estas son delicadas. Esto alargará la vida útil de los sensores.

Se notó que la resistencia del sensor de flexión decrece con su uso.

\section{Recomendaciones}

Se recomienda limitar el movimiento y la velocidad de los servos a fin de no dañar el brazo, además de poner topes contra rotaciones que excedan lo diseñado.

Para el control realimentado de los servomotores de los dedos es recomendable usar sensores FSR, a fin de tener una medida de fuerza de agarre y no destruir o dañar el objeto a manipular.

Dependiendo de la calidad de la impresión, se tendrá que pulir las piezas para recubrir el brazo con barniz para mayor duración y evitar que se rompa.

Se debe calibrar al sensor de flexión de acuerdo a su tiempo de uso.

\section{REFERENCIAS}

[1] Servicio de Información y Noticias Científicas. (2012). "Las sustancias químicas peligrosas causan más muertes que los accidentes de trabajo". España.

[2] Gonzales, A. \& Duran, C. 2009. "El TMR-1. Un robot móvil teleoperado". Revista Colombiana de tecnologías avanzadas. Colombia.

[3] Wabesen, R. "Bluetooth HC-06 Datasheet", consultado de: http://www.mcu-turkey.com/wpcontent/uploads/2013/01/HC-Serial-Bluetooth-Products-201104.pdf

[4] Microchip Data Sheet PIC16F87XA 28/40/44-Pin Enhanced Flash Microcontrollers.

[5] http://vegard.hammerseth.com/2009/03/school-project-mechanical-hand/395/

[6] http://robots-rgentina.com.ar/Actuadores_manos.html

[7] http://inmoov.blogspot.com/

[8] Izquierdo, M. 2008. "Biomecánica y bases neuromusculares de la actividad física y el deporte", Médica Panamericana.

[9] Comité Español de Automática. 2008. "Libro blanco de la robótica», CEA-GTRob. España.

[10] Spectra Simbol, "Flex sensor Datasheet", consultado de: https://www.sparkfun.com/datasheets/ Sensors/Flex/FLEXSENSOR.pdf

[11] Stephen, H. 2012. "Electronics for Electricians”, Cengage Learning, USA.

[12] Pallás, R. Sensores y Acondicionadores de Señal. Alfaomega, 2007.

[13] Abbott, R. "PIC C Compiler Developments", consultado de: http://www.fored.co.uk/html/ Manual.pdf. Junio 2010.

[14] Rafael Enríquez H. 2009. “Arduino User Manual”, Descargado de: http://creativecommons.org/ licenses/by-nc-a/3.0/.CreativeCommon.

[15] Michael Margolis, "Arduino Cookbook”. O’Reilly Media, 2011.

[16] Jazar, R. 2010. "Theory of Applied Robotics", Springer, USA.

[17] Ogata, K. 2010. "Ingeniería de Control Moderna", Pearson Education, México. 